\title{
System Wide Trust: A Possible Contagion Effect
}

\author{
Rian Mehta and Stephen Rice \\ Florida Institute of Technology
}

\begin{abstract}
System Wide Trust (SWT) theory states that when dealing with independent components of one systems, a failure in one component can have a negative effect on the person's trust in other components. The purpose of this study was to examine the extent of the declining trust and whether the failure of an automated aid on board a commercial airline flight would have a contagion effect on the trust in other aids and human entities. The study included 392 participants from the United States and India, who were presented with a hypothetical scenario wherein the automated system that operates the oxygen masks on a commercial flight had failed. Participants were asked to rate their trust in the failed automated aid, as well as four other independent devices/aids on board the aircraft. They were then asked to rate their trust in the pilot, co-pilot, flight attendants, the maintenance manager, and the CEO of the airline. The SWT effect as well as the contagion effect was found to exist for both countries of origin thus suggesting that passengers treat all the automated aids and human entities involved in a commercial airline flight as part of one system. The cross cultural analysis between the two nationalities showed that American participants were more extreme in their responses under both scenarios as compared to their Indian counterparts. Additionally, a mediation analysis revealed that affect (emotion) was a mediating factor in the relationship between the condition and the trust ratings.
\end{abstract}

\section{Introduction}

Automation has increasingly become part of everyday life over the past two decades. This is especially true for the commercial aviation industry. With the increased usage of automation over the years, has also come an increase in the reliance or trust in these systems to perform accurately and effectively over and over again (Dzindolet, Peterson, Pomranky, Pierce, \& Beck, 2003; Parasuraman \& Riley, 1997). There are many facets to the complex relationship between trust and automation. One realm of this field of research has involved System Wide Trust (SWT) Theory. SWT theory suggests that an operator tends assign a single level of trust in a system as a whole rather than in the independent aids, and therefore treats a group of automated aids as one system (Geels-Blair, Rice, \& Schwark, 2013; Keller \& Rice, 2010; Rice \& Geels, 2010). The focus of previous SWT studies has been on automated aids. For the purpose of this study aids, and automated aids refers to devices or technological systems the assist or are necessary components in the overall task of operating a flight. This study seeks to determine the presence of one possible contagion effect, wherein trust in human entities declines due to the presence of a failure in an automated aid. The study will also conduct a cross-cultural analysis to determine if the effects vary as a function of country of origin by utilizing participants from both India and the United States of America. Lastly, the study will perform a mediation analysis using affect as a potential mediator to determine whether participants are basing their decisions on emotions. 


\section{Trust and Automation}

Trust can be defined in different ways, depending on the context of the research setting. The definition of trust that is most apt for this research is the one put forth by previous studies (Deutsch, 1958; Eckel \& Wilson, 2004; Ergeneli, Saglam, \& Metin, 2007) which states that trust is the predictability of another person. Similarly, Meyer, et. al (1995) stated that trust could be explained in the form of vulnerability and relinquishment of control to another person or object. Lastly, trust has also been defined as being dependent on the performance of an object or an individual and the faith in the same to perform what is expected (Barber, 1983; Rempel et al., 1985; Rotter, 1967). The similarities in all the previous research lies in the idea of defining trust as a psychological construct or emotion based on faith and dependence in what is expected of another person or object. For the purpose of this study, the Meyer, et. al (1995) definition of trust is most appropriate.

Trust, while being a strong psychological construct, can be volatile. Once trust in a person, object or system is lost, it can be difficult to regain. Slovic (1993) claimed that if trust is lost it might never be recovered. This shows the importance of trust in any industry, especially one such as aviation. Trust is crucial in so many of the relationships involved in aviation such as the human-human trust between the passengers and the pilots, the human-automation relationship between the pilots and the automation, and many more.

Automation has begun to take leading role in the operation of commercial airline flights, and therefore understanding the relationship between trust and the automation is of significance to the research industry. Studies have shown that the perception of reliability significantly affects a person' trust in the automation (Lee \& See, 2004; Geels-Blair, Rice, \& Schwark, 2013; Wiegmann, Rich \& Zhang, 2001; Parasuraman \& Riley, 1997; Rice, 2009; Muir, 1994; Rice \& Geels, 2010; Muir, \& Moray, 1996). While human-to-human trust has been defined as a psychological construct, Geels-Blair, Rice and Schwark (2013) stated that the same idea of trust could be applied to the trust relationship between the human and the machine. Additionally, Rice (2009) showed a positive relationship between reliance and trust, whereby operators were more trusting when they were able to predict the outcome of the automation.

\section{Cultural Considerations}

Aviation being a universal industry around the world, it is important to understand how consumers from different countries and cultures perceive different situations. Culture has been defined as "a set of shared values and beliefs that characterize national, ethnic, moral and other group behavior" (Faure and Sjostedt 1993; Craig and Douglas 2006; Adapa 2008). For the purpose of this research, participants from the United States of America and India were used to determine the presence of any differences between how consumers felt towards the situation.

While these two nationalities represent participants from two widely separated geographic regions, each also represents a different type of cultural society. Hofstede $(1980,2001)$ stated that the Indian culture was collectivistic in nature. India scored a 48 out of a 100 on the individualistic versus collectivist dimension on Hofstede's Cultural Values Index (Robbins \& Judge, 2009). Similarly, The United States scored a 91 out of 100 on the same index. Rice, et. al (2014) stated, however, that due to the median score of 48, Indian culture may exhibit some individualistic tendencies even though being collectivistic in general. Markus and Kitayama (1991) explained that in a collectivistic society citizens are considered to be interdependent on one another, while in an individualistic culture such as the United States, citizens tend to be more independent. 
Citizens of individualistic cultures are taught to not trust without questioning, and to always be independent, while those of collectivistic societies are taught to never question authority and encouraged to always trust (Han, 1994; Wu \& Jang, 2008). Trubisky, et. al. (1991) also stated that individualists are more confrontational in nature while collectivists tend to be more avoiding and obliging.

Individualistic cultures lay an emphasis on an individual's autonomy and independence (Bochner, 1994; Kashima \& Callan, 1994), while collectivists tend to be focused on the well-being of others and the best interests of the community. These cultural differences do have an impact on the mindset of consumers from different countries and therefore do have an impact on the way they trust. Collectivistic citizens are more likely to trust other people than their individualistic counterparts (Hofstede, 1980). These cultural differences are therefore important to research and understand as they may have an impact on different consumers' propensity to trust, and may therefore provide plausible explanations for the differences in the participants' feelings towards the scenarios.

Previous studies that have employed similar cross-cultural analyses have shown that while participants from both India and the United States shared many similar points of view, American participants were more dramatic in their responses as compared to their Indian counterparts. These differences could be related to the participants' different mindsets when dealing with uncertain situations such as automation failures. Since collectivists have a higher tendency to trust, this could be inferred as a form of taking more risks in uncertain situations such as automation failures. The aim of this study is to see if these differences continue to exist between the two cultures and if they continue to have similar impacts on the participants' level of trust. Passengers in commercial air travel are from around the world, and so it is important to research the differences in passengers' mindsets from all across the globe.

\section{Affect}

Affect, or emotion, is considered to bring about some variance and unpredictability when dealing with a person's decision-making process (Bechara, 2004). While this may not necessarily be a negative aspect, it is important to research and understand the mindset of the participant, which may lead to providing the research with valuable context and plausible explanations as to what impacts the consumers' decisions. Alpert and Rosen (1990) suggested that affect can have several different meanings and interpretations of emotions based on the situation. Affect has been described by psychologists as a set of dimensions that include displeasure, distress, depression, excitement, and many more (Russell, 1980). Similarly, it is important to note that Lewis and Wiegert (1985) stated that interpersonal trust has cognitive and affective foundations. Russell (2003) went on to state that at the heart of emtions are "core affect" states of feeling simply good or bad, and these states can influence reflexes, perception, cognition, and behavior. Lastly, affect has also been analyzed from a geographical perspective to understand its differences and influences around the globe (Thien, 2005).

Affect has been used in previous studies to determine whether it is a possible mediator for the consumers' perceptions of the scenario (Campbell, 2007; Winter, Rice, \& Mehta, 2014; Baker \& Cameron, 1996; Rice, Winter, Kraemer, Mehta, \& Oyman, in press; Babin, \& Attaway, 2000). These studies have shown the influence emotions have on decision making, whereby affect often times mediates the relationship between the condition and the effect. Previous sections have laid out the relationship between trust and automation, where trust is deemed to be a psychological construct. Being a psychological construct based on past experiences and memories, trust will also be influenced by emotions. For the context of this research setting, it is appropriate to trust as an affect-based construct. Affect-based trust has been defined by McAllister (1995) as a type of trust in which emotional ties between individuals are created by sincere concern and support. The 
purpose of the mediation analysis in this research context is to determine whether affect mediates the effect of the SWT theory and the possible contagion effect as well. If affect were found to mediate the relationship, it would allow for a plausible explanation that emotions played a hand in the participants' decline in trust in the

overall system of automated aids and human entities due to a failure at an individual level of one automated aid.

\section{Current Study}

The current study seeks to expand the SWT line of research. Previous studies have researched the effect by utilizing operators and consumers in different forms of systems (Keller \& Rice, 2009). While some of these studies have been involved in the field of aviation automation (Geels-Blair, Rice, \& Schwark, 2013), this study is the first to analyze to possibility of a contagion effect from automation to humans. The study seeks to determine whether there is transference of feelings or changes $\mathrm{n}$ trust levels towards human entities when there is an automation failure on board a commercial airline flight.

Similar to previous SWT studies, participants will be given a hypothetical scenario of a commercial airline flight. They were then asked to rate their feelings and levels of trust in a number of automated aids and human entities. In the context of this research setting, the commercial airline flight with the automation and the human operators all together will be considered as the system. The automated aids involved are the oxygen masks, the auto-pilot system, the airplane's flaps, the landing gear, and the video screens on the backs of the seats. The human entities are the pilot, the co-pilot, the flight attendants, mechanics, and airline CEO. The study utilizes participants from the United States and India in order to conduct a cross-cultural analysis to determine whether differences exist between consumers from different geographic regions. Lastly, the study utilizes the affect data in order to conduct a mediation analysis to determine if affect is in fact a mediator between the condition and the effect. There is valuable insight to be gained from determining if the effect is based on the participants' emotions. The hypotheses for the current study were as follows:

H1: In the failure condition, there will be a drop in trust in the unrelated automated aids or human entities compared to the non-failure condition.

H2: There will be a difference in trust and affect ratings for the unrelated automated aids or human entities as a function of country of origin.

H3: The relationship between the condition and trust will be mediated by affect.

H4: There will be an interaction between the independent variables. However, this is a non-directional prediction, as we have no a priori basis for a directional prediction. 


\section{Methods}

The study includes a multistage process that includes a a two way factorial ANOVA, a three way ANOVA, and a mediation analysis. These aspects are elaborated at each stage.

Participants. Three hundred ninety-two (131 females) participants from India and the United States participated in the study. There were equal amounts of participants from each country. The mean age was 32.30 (SD $=$ 9.87).

Materials and Stimuli. The study was conducted using an online instrument developed with FluidSurveys ${ }^{\circledR}$. Participants were recruited via Amazon’s ${ }^{\circledR}$ Mechanical Turk ${ }^{\circledR}$ (MTurk). MTurk provides participants who complete human intelligence tasks in exchange for monetary compensation. Prior research shows that data from MTurk is as reliable as normal laboratory data (Buhrmester, Kwang, \& Gosling, 2011; Germine, et al., 2012). Participants were asked to fill out a consent form and then given instructions. All participation was voluntary and anonymous through MTurk, and all participants were compensated for the completion of the survey.

Procedure. Participants were presented with a hypothetical scenario of being on board a commercial airline flight. There were two versions of the questionnaire, one containing a failure of an automated aid (failure condition), and one containing no failure (control condition). Each of the hypothetical scenarios and their corresponding questions are shown in Appendix A. In the failure condition, the automated aid that fails was the oxygen masks. The participants were randomly assigned to either of the groups.

The survey asked participants to rate their feelings of trust in various different automated aids, and human entities. The ratings were measured on a Likert-type scale from -3 (extremely distrust) to +3 (extremely trust) with a neutral option of zero (neither trust nor distrust). Affect was measured using three questions on the instrument to gauging how the participants feel about the scenario. The questions presented to the participants are shown in Appendix B. Lastly, participants were asked for demographic information, debriefed and dismissed.

\section{Design}

Stage 1. For the set of analyses, the study utilizes an experimental factorial design. First, a two-way factorial ANOVA was conducted on the affect data to understand the differences between the affect ratings of the participants from India and the United States. The independent variables were Failure/Non Failure of the automation, and Country of Origin. Secondly, a three-way factorial ANOVA using a 2x2x10 design was conducted on the trust data. The three independent variables being measured in this study were Failure/Non Failure of the automation, Country of Origin, and type of automated device or category of human entity. The dependent variable was trust.

Stage 2. The initial analysis performed a three-way ANOVA which included all five automated aids and five human entities including the oxygen masks. The oxygen mask was the automated aid that was said to have failed and so it is expected to experience a lower level of trust from the participant. For this reason, a second analysis was performed. For this analysis the scored for the four remaining automated aid were averaged into one rating, and the scores of the five human entities were averaged into another. This stage utilized a three-way factorial ANOVA with a 2x2x2 design. The three independent variables being measured were still Failure/Non Failure of the automation, Country of Origin, and type of automated device or category of human entity. 
However, the levels of the last IV were the average ratings of the automated aids, and the average ratings of the human entities. Trust remained the dependent variable.

Stage 3. The final analysis conducted in this study was a mediation analysis to determine whether affect was a possible mediator for the effect. The mediation analysis was conducted for participants from both countries to compare the failure condition to the non-failure condition with respect to their feelings towards the automated aids and human entities. For this stage in the analysis, the oxygen mask ratings were excluded as well.

For all the analyses in the three stages, the scale of measurement for both DVs (trust and affect) is ordinal, but the data was treated as an interval scale of measurement. This assumption was made as values of equal magnitude difference were assigned to each response of the Likert type scale (Göb, McCollin, and Ramalhoto, 2007).

\section{Results}

\section{Stage 1}

To produce a single value describing the participant's overall trust in the situation, all the values of the trust questions were averaged into one. The same was done for the affect data. A Cronbach's Alpha test was conducted for each as a measure of internal consistency. A two-way ANOVA was conducted on the affect data, with Failure/Non Failure of the automation, and Country of Origin of the participants as the factors. There was a main effect of Failure, $F(1,388)=225.610, p<.001$, partial-eta squared $=0.371$. There was a main effect of Country, $F(1,388)=6.203$, $p=.001$, partial-eta squared $=0.022$. These effects were qualified by a significant interaction between Failure and Country, $F(1,388)=11.960, p<.001$, partial-eta squared $=.003$. This suggests that the American participants were more extreme in their views towards failure condition as compared to their Indian counterparts. Figure 1 displays the affect data for both participant groups in both conditions.

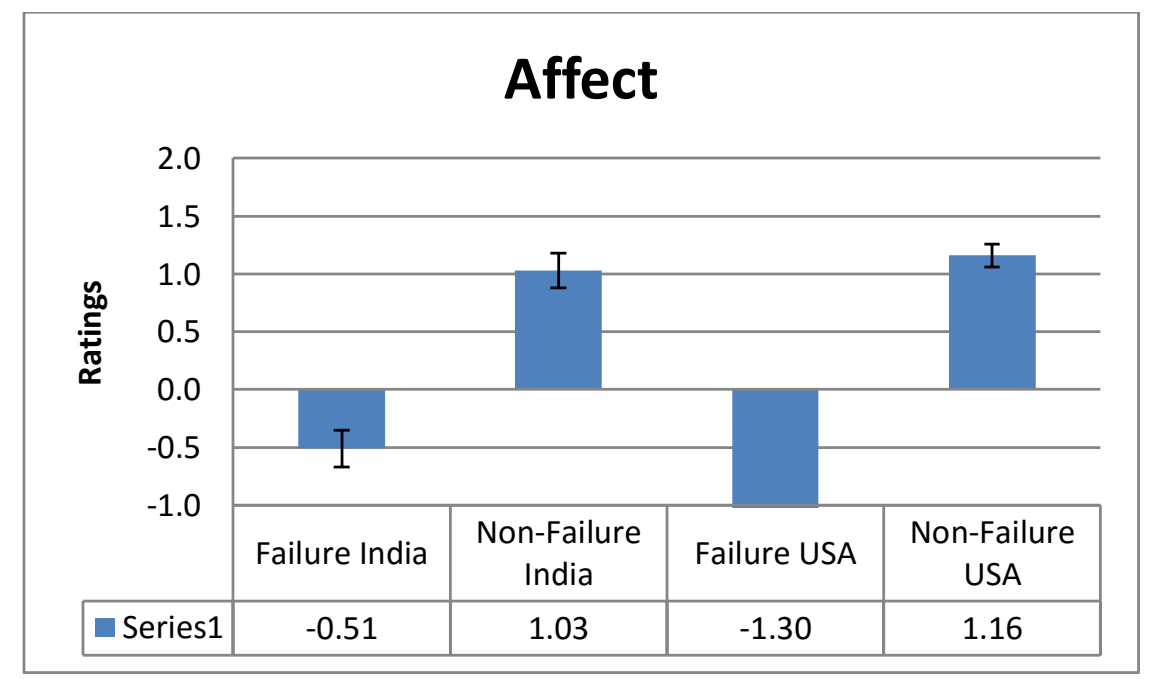

Figure 1. Affect Data for Indian and U.S. participants for Failure and Non- Failure. 
A 2x2x10 ANOVA was conducted on the Trust data, with Type of automated device or category of human entity, Failure/Non Failure of the automation, and Country of Origin of the participants as the factors. There was a main effect of Failure, $F(1,388)=104.878, p<.001$, partial-eta squared $=.213$. There were no other significant effects. There was a main effect of Type of automated device or category of human entity, $F(9,388)$ $=48.459, p<.001$, partial-eta squared $=.111$; however, this effect was qualified by three significant interactions. The first was between items and country, $F(9,388)=12.621, p<.001$, partial-eta squared $=.032$. The second was between items and failure, $F(9,388)=28.571, p<.001$, partial- eta squared $=.069$. The final interaction was a three way interaction between items, country and failure, $F(9,388)=6.660, p<.001$, partial-eta squared $=.017$. Participants showed a significant decline in trust in both human entities and automated aids, suggesting the presence of SWT effect and a contagion effect. The trust data for the Indian and American participants are shown below in Figure 2 and Figure 3 respectively.

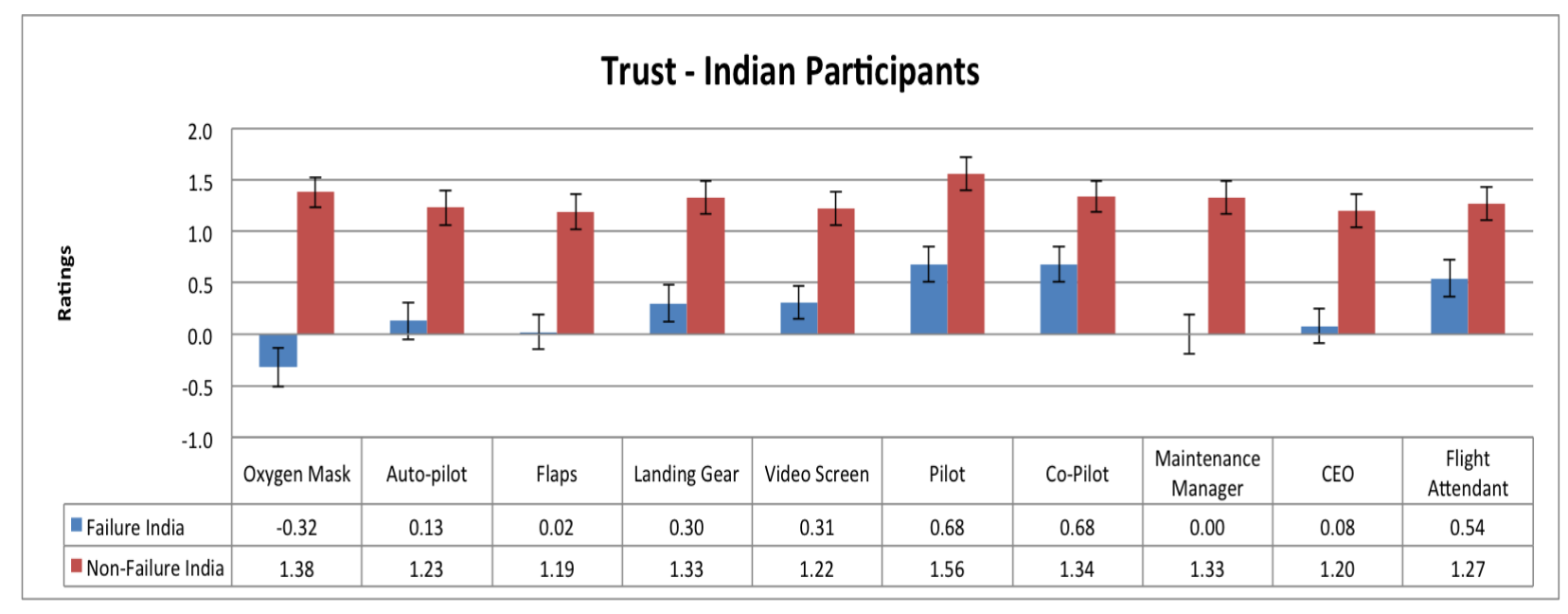

Figure 2. Trust Data for Indian participants for Failure and Non-Failure Conditions.

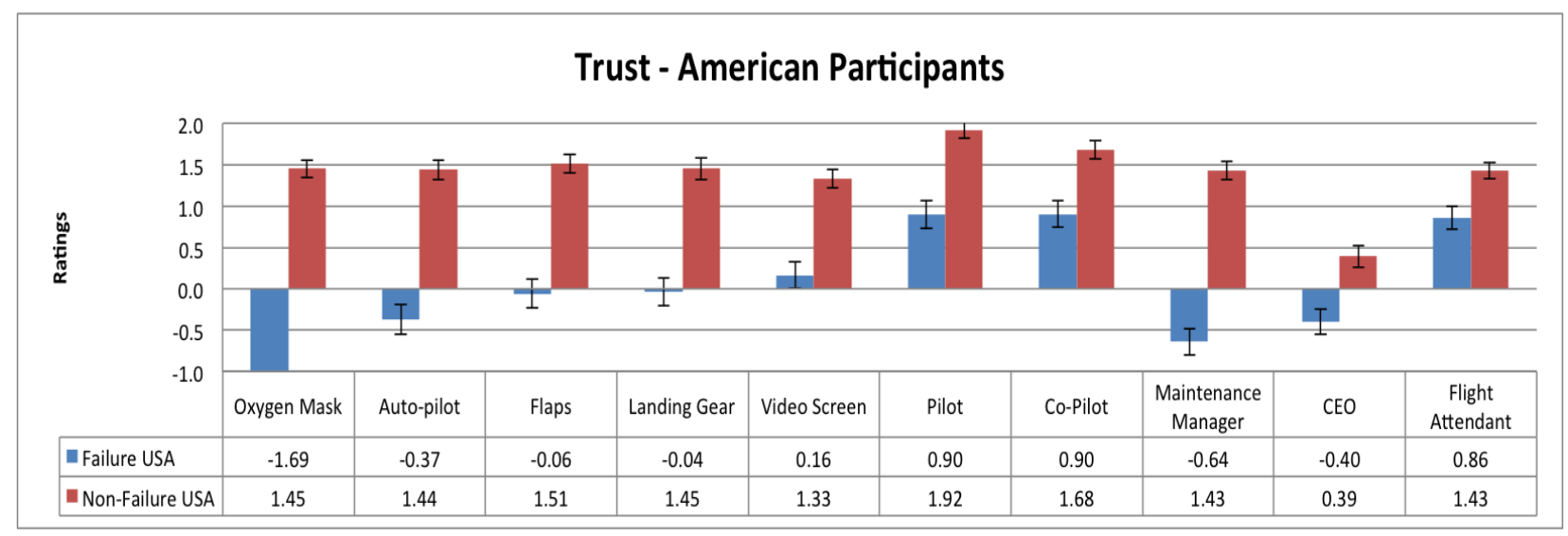

Figure 3. Trust Data for American participants for Failure and Non-Failure Conditions. 


\section{Stage 2}

A 2x2x2 ANOVA was conducted on the Trust data, with Type of automated device or category of human entity, Failure/Non Failure of the automation, and Country of Origin of the participants as the factors. There was a main effect of Failure, $F(1,388)=84.333, p<.001$, partial-eta squared $=.179$. There were no other significant effects. There was a main effect of Type of automated device or category of human entity, $F(1,388)$ $=15.231, p<.001$, partial-eta squared $=.038$; however, this effect was qualified by two significant interactions. The first was between items and failure, $F(1,388)=12.326, p<.001$, partial- eta squared $=.031$. The second interaction was a three way interaction between items, country and failure, $F(1,388)=4.432, p<.001$, partialeta squared $=.011$. This analysis suggested that the drop in trust was still significant indicating the presence of the SW'T effect and contagion effect with the effect of the oxygen masks removed from the analysis. Figure 4 shows the trust averages for both the Indian and American participants on the four automated aids and the five human entities.

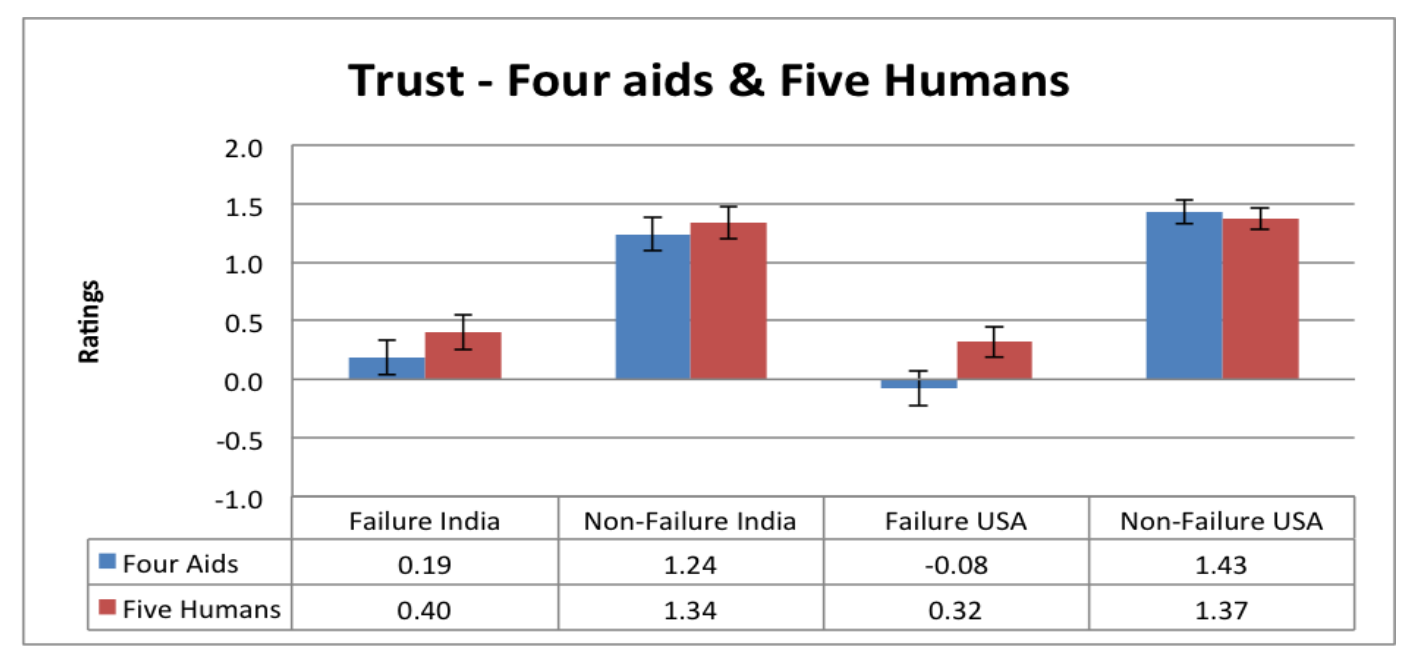

Figure 4. Trust data on the four automated aids and the five human entities

\section{Stage 3}

The first mediation analysis was conducted using Indian participants to compare the failure condition to the non-failure condition with respect to their feelings towards the automated aids. The paths for this mediation analyses can be found in Figure 5A. In order to conduct the mediation analysis, the correlation between Condition and Trust was first found to be significant, $r=-.349, p<.001$, showing that the initial variable correlated with the outcome variable. The standardized path coefficients were: condition to affect $(\beta=$ $-.447, p<.001)$; affect to trust $(\beta=.383, p<.001)$; condition to trust controlling for affect $(\beta=-.178 ; p=.012)$. These data show that Affect had a partial mediating effect on the relationship between Condition and Trust.

The second mediation analysis was conducted using Indian participants to compare the failure condition to the non-failure condition with respect to their feelings towards the human entities. The paths for this mediation analyses can be found in Figure 5B. In order to conduct the mediation analysis, the correlation 
between Condition and Trust was first found to be significant, $r=-.318, p<.001$, showing that the initial variable correlated with the outcome variable. The standardized path coefficients were: condition to affect $(\beta=$ $-.447, p<.001)$; affect to trust $(\beta=.404, p<.001)$; condition to trust controlling for affect $(\beta=-.138 ; p=.052)$. These data show that Affect completely mediated the relationship between Condition and Trust.

The third mediation analysis was conducted using American participants to compare the failure condition to the non-failure condition with respect to their feelings towards the automated aids. The paths for this mediation analyses can be found in Figure 5C. In order to conduct the mediation analysis, the correlation between Condition and Trust was first found to be significant, $r=-.524, p<.001$, showing that the initial variable correlated with the outcome variable. The standardized path coefficients were: condition to affect $(\beta=$ $-.766, p<.001)$; affect to trust $(\beta=.515, p<.001)$; condition to trust controlling for affect $(\beta=. .130 ; p=.142)$. These data show that Affect completely mediated the relationship between Condition and Trust.

The fourth mediation analysis was conducted using American participants to compare the failure condition to the non-failure condition with respect to their feelings towards the human entities. The paths for this mediation analyses can be found in Figure 5D. In order to conduct the mediation analysis, the correlation between Condition and Trust was first found to be significant, $r=-.441, p<.001$, showing that the initial variable correlated with the outcome variable. The standardized path coefficients were: condition to affect $(\beta=$ $-.766, p<.001)$; affect to trust $(\beta=.647, p<.001)$; condition to trust controlling for affect $(\beta=.054 ; p=.544)$. These data show that Affect completely mediated the relationship between Condition and Trust.

A

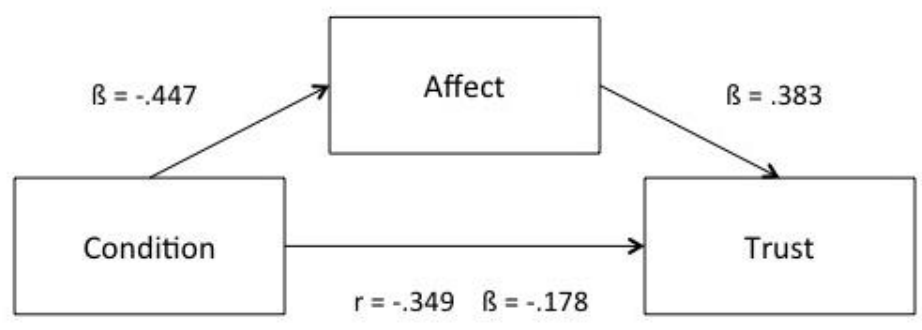

B

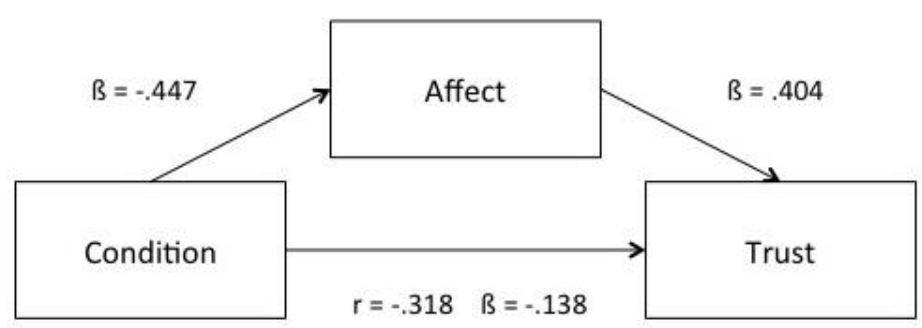

Figure 5. Paths for the mediation analyses 

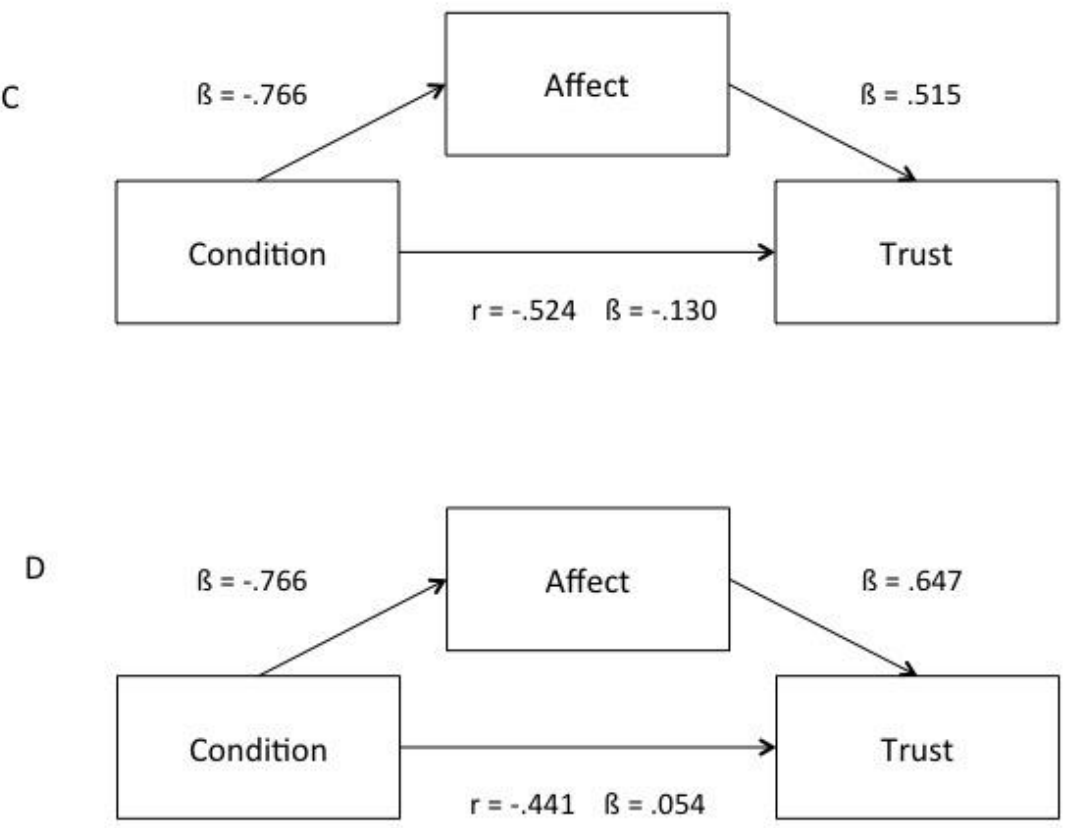

Figure 5 [continued]. Paths for the mediation analyses

\section{Discussion}

The aim of this study was to further research SWT theory and explores the possibilities of one possible contagion effect. Additionally, this research sought to analyze the cultural differences between two different countries of origins, to see whether they had an impact on the SWT effect. The findings of the study support the research predictions for the most part.

The first hypothesis predicted that participants would have reduced levels of trust in the automated aids and human entities when the oxygen masks were said to have failed. The findings of the data analysis supported this hypothesis, and therefore suggest the presence of the contagion effect. This was found to be true for in both stages of the data analysis. Stage 2 attempted to remove the effect of the failed aid, the oxygen mask, and determine whether the SWT contagion effect was still statistically significant. Once an automated aid failed, the participants' trust in other unrelated aids and unrelated human entities decreased as well, supporting the theory of the presence of a contagion effect. One possible explanation for the same is that consumers consider all parts of a commercial airline operation, i.e. automated aids and human entities, as one large system. Failure in one part of the system negatively affects trust levels in several other parts of the system. The results of the study showed a decrease in trust in the airline CEO. This relationship is interesting to note. Tsui, Zhang, Wang, Xin, and Wu (2006) suggest that there is a direct relationship between the values, leadership and behavior of a CEO and the organizational culture. This could suggest that passengers believe the CEO to be ultimately responsible for the safety culture of the airline, and is therefore indirectly responsible for all potential dangerous occurrences.

The second research prediction stated that there would be differences in trust ratings based on country of origin. The data was collected from participants in India and the United States in order to perform the cross- 
cultural analysis. As mentioned earlier, prior research has suggested that these two countries represent two different cultures and mindsets. India is a said to be a more collectivistic society with the focus being placed on the well-being of others and the community as a whole. On the other hand, the United States is said to be a more individualistic society, which emphasizes placing concern for one above all else. The results of the study supported the research prediction, where significant differences were identified between the trust ratings of participants from each country. The data suggested that American participants were more extreme in their responses as compared to their Indian counterparts. The American participants were more trusting in control condition and less trusting in the failure condition as compared to the Indian counterparts. One plausible explanation for the same could be the differences in cultural upbringing and the differences in mindsets of participants from these different cultures. Since citizens of collectivistic cultures are taught to trust without question, their viewpoints may be different from those brought up in individualistic societies.

The third hypothesis stated that affect would mediate the relationship between the condition and trust. As stated earlier, affect refers to the participants' emotion which may be an influencer in the decision making process. The mediation analysis suggested that affect did mediate the relationship, thereby suggesting that the participants were basing their decisions of trust on their emotions. While emotions are not necessarily a negative aspect of decision-making, they do provide a fair amount of variance and unpredictability. The results of the mediation analysis additionally showed that affect mediated the relationship for both Indian and American participants. This suggests that even though differences in trust ratings was observed between the participants of these two countries, they were both basing their decisions on emotions. The findings of this study align with the results of previous research on the fact that affect was found to mediate the relationships between participants' trust and the scenarios. This adds to the scientific research on the decision-making mindset of aviation consumers.

Lastly, the study predicted that there would be an interaction between the variables. The results supported this hypothesis as the main effect was qualified by three significant interactions. These interactions were between items and country, items and failure, and a three-way interaction between items, country and failure.

\section{Limitations and Practical Implications}

All research studies, including this one, are subject to some limitations. The limitations for this study have been identified and must be kept in mind when analyzing the results and interpreting the findings of the same. The most salient limitation of this study is data collection technique and methodology used. Participants for the study were recruited via Amazon's ${ }^{\circledR}$ Mechanical Turk $\mathbb{~}($ MTurk). The issue with such a data collection tool is that the researcher is not in complete control and unable to supervise the testing environment. The convenience and ability of collecting large samples makes it necessary accept this limitation as part of the study. Buhrmester, Kwang and Gosling (2011) and Germine, et al. (2012) stated that data collected from MTurk is as reliable as laboratory data.

While the data collection method does allow for a fairly large convenient sample, the generalizability of the results is limited. Due to the fact that participants were used from only two countries, the study is able to perform a cross-cultural analysis, however the findings cannot be generalized to apply to aviation in all the countries across the globe. Similarly, the study does not discriminate against persons that have never flown on a commercial airline flight before. This is a limitation when attempting to interpret these findings as those of aviation consumers. A participant may not in fact be an aviation consumer, but may be allowed to participate in 
the study. When attempting to conduct such a complex study design, a large sample size is required and in order to achieve that, certain limitation must be accepted.

While there are certain limitations to the study, the research does have partial implications on the aviation industry. Research in any field provides a better understanding of the industry. System Wide Trust has been research in previous studies but this research suggests the presence of the contagion effect of decreased trust, due to failed automation, in human entities. This is useful information to be aware of in any commercial aviation setting. Aviation is an essential mode of travel, but airlines are always in heavy competition, and therefore customer service and satisfaction is a big priority for all air service operators to ensure loyal customers and sustain revenue flow. This study shows that due to a negative experience, passengers may experience a system wide contagion effect that could decrease their trust in the CEO, which in turn could decrease their trust in that particular airline as a whole. This may motivate a passenger to choose to fly on a different airline on following journeys. In this manner it is entirely possible that the contagion effect could have significant financial implications for an airline, and therefore this research is of value and importance to the industry by providing a better understanding of the mindset of the passengers.

Another possible practical implication of this research is that if a SWT contagion effect was found in aviation, it could suggest that it is also present in other industries. This could lead to important research leading to a more in depth understanding of several other fields as well. Lastly, since this study is a continuation of the line of research involving SWT, this study reinforces the foundation of the theory, which is of importance to the scientific community.

\section{Future Research}

There are several research opportunities that still remain in this new line of research involving SWT. Previous studies in this field have mainly been centered on the aviation industry. While this is useful to the understanding of passenger mindsets, there may be several other applications for this theory in other fields and industries. Future research could use this study as a foundation upon which to analyze the mindset of customers in other consumer-oriented fields, and could additionally research whether the contagion effect does exist as well.

The main goal of future studies should be to focus on the aforementioned limitations of the current research. Future research should seek to replicate the study using participants from different countries, in order to gain a more globally representative analysis of the effect. It would be interesting to study how the SWT effect varies as a function of country of origin.

\section{Conclusion}

The aim of the study was to explore the System Wide Trust theory deeper in order to determine the presence of a possible contagion effect. The results of the data analysis suggested that passengers' trust in several different human entities and automated aids were negatively affected by the presence of one failed automated aid. The practical implications are of importance to the aviation industry in an effort to better understand the mindset of the travelling public. However, the secondary layer of the study showed the differences in trust ratings of passengers from different cultures, i.e. two different countries of origin. The cross-cultural analysis provides global perspective of the difference existent within the aviation industry. This study provides a platform for future research to further examine these psychological impacts of trust within aviation and other industries as well. SWT has been researched in several different studies, mainly with respect 
to the aviation industry, but with the expansion of the theory in terms of a possible contagion effect, future research may begin to apply the theory in several other industries.

\section{References}

Adapa, S. (2008). Adoption of internet shopping: Cultural considerations in India and Australia, Journal of Internet Banking and Commerce, 13(2), 1-17.

Alpert, M., \& Rosen, A. (1990). A semantic analysis of the various ways that the terms "affect,"“'emotion," and "mood" are used. Journal of communication disorders, 23(4), 237-246.

Babin, B. J., \& Attaway, J. S. (2000). Atmospheric affect as a tool for creating value and gaining share of customer. Journal of Business research, 49(2), 91-99.

Baker, J., \& Cameron, M. (1996). The effects of the service environment on affect and consumer perception of waiting time: An integrative review and research propositions. Journal of the Academy of Marketing Science, 24(4), 338-349.

Barber, B. (1983). The logic and limits of trust (Vol. 96). New Brunswick, NJ: Rutgers University Press.

Bechara, A. (2004). The role of emotion in decision-making: evidence from neurological patients with orbitofrontal damage. Brain and cognition, 55(1), 30-40.

Bochner, S. (1994). Cross-Cultural Differences in the self concept a test of Hofstede's individualism/collectivism distinction. Journal of cross-cultural psychology, 25(2), 273-283.

Buhrmester, M., Kwang, T., \& Gosling, S. D. (2011). Amazon’s Mechanical Turk: A new source of inexpensive, yet high-quality data? Perspectives on Psychological Science, 6(3), 3-5.

Campbell, M. C. (2007). “Says who?!” How the source of price information and affect influence perceived price (un) fairness. Journal of Marketing Research,44(2), 261-271.

Craig, C. S., \& Douglas, S. P. (2006). Beyond national culture: implications of cultural dynamics for consumer research, International Marketing Review, 26(3), 322-342.

Deutsch, M. (1958). Trust and suspicion. The Journal of Conflict Resolution, 2, 265-279.

Dzindolet, M. T., Peterson, S. A., Pomranky, R. A., Pierce, L. G., \& Beck, H. P. (2003). The role of trust in automation reliance. International Journal of Human-Computer Studies, 58(6), 697-718.

Eckel, C. C. \& Wilson, R. K. (2004). Is trust a risky decision? Journal of Economic Behavior \& Organization, 55, 447465.

Ergeneli, A., Saglam, G., \& Metin, S. (2007). Psychological empowerment and its relationship to trust in immediate managers. Journal of Business Research, 60, 41-49. 
Faure, G. O. \& Sjostedt, G. (1993). Culture and negotiation: An introduction, Newbury Park: Sage Publications.

Geels-Blair, K., Rice, S., \& Schwark, J. (2013). Using system-wide trust theory to reveal the contagion effects of automation false alarms and misses on compliance and reliance in a simulated aviation task. The International Journal of Aviation Psychology, 23(3), 245-266, DOI: 10.1080/10508414.2013.799355

Germine, L., Nakayama, K., Duchaine, B.C., Chabris, C.F., Chatterjee, G., \& Wilmer, J.B. (2012) Is the web as good as the lab? Comparable performance from web and lab in cognitive/perceptual experiments. Psychonomic Bulletin \& Review, 19(5), 847-857.

Göb, R., McCollin, C., \& Ramalhoto, M. F. (2007). Ordinal methodology in the analysis of Likert scales. Quality \& Quantity, 41(5), 601-626.

Han, S. \& Shavitt, S. (1994). Persusion and Culture: Advertising Appeals in Individualistic and Collectivistic Societies. Journal of Experimental Social Psychology, 30, 326-350.

Hofstede, G. (1980). Motivation, leadership and organization: do American theories apply abroad? Organizational Dynamics, $9(1)$ : 42-63.

Hofstede, G. (2001). Culture 's Consequences: Comparing Values, Behaviors Institutions, and Organizations across Nations - 2nd Edition, Thousand Oaks: Sage Publications.

Kashima, Y., Callan, V. J., Triandis, H. C., Dunnette, M. D., \& Hough, L. M. (Eds.) (1994). Handbook of industrial and organizational psychology. Palo Alto, CA: Consulting Psychologists Press.

Keller, D. \& Rice, S. (2010). System-wide versus component-specific trust using multiple aids. The Journal of General Psychology, 137(1), 114-128.

Lee, J. D., \& See, K. A. (2004). Trust in automation: Designing for appropriate reliance. Human Factors: The Journal of the Human Factors and Ergonomics Society, 46(1), 50-80.

Lewis, J. D., \& Weigert, A. 1985. Trust as a social reality. Social Forces, 63: 967-985.

Markus, H. R. \& Shinobu K. (1991). Culture and the self: Implications for cognition, emotion, and motivation. Psychological Review, 98(2): 224-253.

McAllister, D.J. (1995) Affect- and cognition-based trust as foundations for interpersonal cooperation in organizations. academy of Management Journal, 38, 24-59.

Meyer, J. (2001). Effects of warning validity and proximity on responses to warnings. Human Factors, 43, 563 572.

Meyer, J. (2004). Conceptual issues in the study of dynamic hazard warnings. Human Factors, 46(2), 196-204.

Muir, B. M. (1994). Trust in automation: Part I. Theoretical issues in the study of trust and human intervention in automated systems. Ergonomics, 37(11), 1905-1922. 
Muir, B. M., \& Moray, N. (1996). Trust in automation. Part II. Experimental studies of trust and human intervention in a process control simulation.Ergonomics, 39(3), 429-460.

Parasuraman, R., \& Riley, V. (1997). Humans and automation: Use, misuse, disuse, abuse. Human Factors, 39, 230-253.

Rempel, J. K., Holmes, J. G., \& Zanna, M. P. (1985). Trust in close relationships. Journal of Personality and Social Psychology, 49(1), 95-112. doi:http://dx.doi.org/10.1037/0022- 3514.49.1.95.

Rice, S. (2009). Examining Single- and Multiple-Process Theories of Trust in Automation. Journal Of General Psychology, 136(3), 303-319.

Rice, S. \& Geels, K. (2010). Using system-wide trust theory to make predictions about dependence on four diagnostic aids. The Journal of General Psychology, 137(4), 362-375.

Rice, S., Kraemer, K., Winter, S. R., Mehta, R., Dunbar, V., Rosser, T. G., \& Moore, J. C. (2014). Passengers from India and the United States Have Differential Opinions about Autonomous Auto-Pilots for Commercial Flights. International Journal of Aviation, Aeronautics, and Aerospace, 1(1). Retrieved from http://commons.erau.edu/ijaaa/vol1/iss1/3

Rice, S., Winter, S.R., Kraemer, K., Mehta, R. \& Oyman, K. (in press). How do depression medications taken by pilots affect passengers' willingness to fly? A mediation analysis. Review of European Studies.

Robbins, S. P. \& Judge, T. A. (2009). Organizational behavior (13th Ed.). Upper Saddle River NJ: Prentice Hall.

Rotter, J. B. (1967). A new scale for the measurement of interpersonal trust. Journal of personality, 35(4), 651-665.

Russell, J. A. (1980). A circumplex model of affect. Journal of personality and social psychology, 39(6), 1161.

Russell, J. A. (2003). Core affect and the psychological construction of emotion. Psychological review, 110(1), 145.

Slovic, P. (1993). Perceived Risk, Trust, and Democracy. Risk. Analysis, 13: 675-682. doi: 10.1111/j.15396924.1993.tb01329.x

Thien, D. (2005). After or beyond feeling? A consideration of affect and emotion in geography. Area, 37(4), 450-454.

Trubisky, P., Ting-Toomey, S. and Lin, S.L. (1991). The influence of individualism collectivism and self-monitoring on conflict styles, International Journal of Intercultural Relations, Vol. 15 No. $1,65-84$.

Tsui, A. S., Zhang, Z. X., Wang, H., Xin, K. R., \& Wu, J. B. (2006). Unpacking the relationship between CEO leadership behavior and organizational culture.The Leadership Quarterly, 17(2), 113-137.

Winter, S. R., Rice, S., \& Mehta, R. (2014). Aviation Consumers' Trust in Pilots: A Cognitive or Emotional Function. International Journal of Aviation, Aeronautics, and Aerospace, 1(1). Retrieved from http://commons.erau.edu/ijaaa/vol1/iss1/2 
Wiegmann, D. A., Rich, A., \& Zhang, H. (2001). Automated diagnostic aids: The effects of aid reliability on users' trust and reliance. Theoretical Issues in Ergonomics Science, 2(4), 352-367. 


\title{
Appendices
}

\section{Appendix A - Questions measuring trust}

\section{Failure Condition}

\begin{abstract}
Imagine that you are flying on a 4-hour commercial airplane flight from one major city to another. Sometime during the flight, an alarm goes off throughout the cabin and oxygen masks fall from the compartments above passenger seats. Following this, the pilot comes on the intercom and says that there was a mistake and the automation that operated the oxygen masks failed. He says that there is no actual emergency and not to worry. The pilot then tells you the altitude of flight and how long it will be before you land.
\end{abstract}

Please rate your trust in the automation that operates the oxygen masks.

OEtremely Trust

OQuite Trust

SIllghtly Trust

Neither Trust nor Distrust

OSlightly Distrust

Quite Distrust

Extremely Distrust

Please rate your trust in the automation that operates the airplane's auto-pilot system.

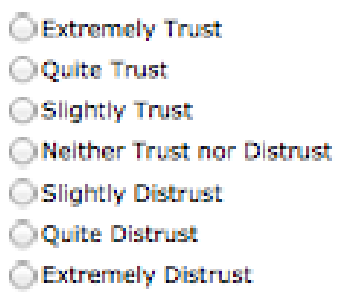

Please rate your trust in the automation that operates the airplane's flaps.

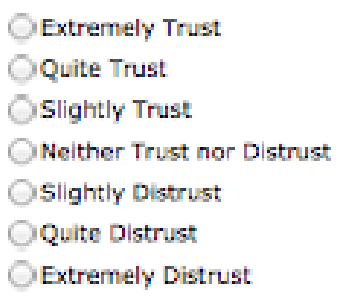

Please rate your trust in the automation that operates the airplane's landing gear.

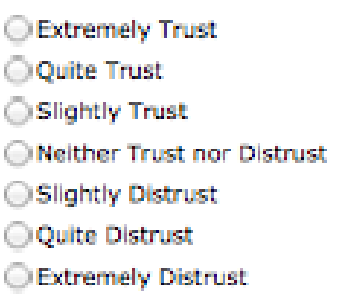


Please rate your trust in the automation that operates the airplane's video screens on the backs of the seats.

OExtremely Trust

QQuite Trust

OSlightly Trust

ONeither Trust nor Distrus:

OSlightly Distrust

QQuite Distrust

OExtremely Distrust

Please rate your trust in the pilot that operates the airplane.

OExtremely Trust

QQuite Trust

OSlightly Trust

ONeither Trust nor Distrus:

Oslighty Distrust

OQuite Distrust.

OExtremely Distrust

Please rate your trust in the co-pilet that operates the airplane.

OExtremely Trust

OQuite Trust

OSlighty Trust

ONeither Trust nor Distrus:

Oslightly Distrust

QQuite Distrus:

OExtremely Distrust

Please rate your trust in the maintenance manager for the airline.

OExtremely Trust

OQuite Trust

Oslightly Trust

ONeither Trust nor Distrus:

OSlightly Distrust

Quite Distrus:

OExtremely Distrust

Please rate your trust in the CEO of the airline manufacturer.

OExtremely Trust

OQuite Trust

OSlightly Trust

ONeither Trust nor Distrust

Oslightly Distrust

Quite Distrust

OExtremely Distrust

Please rate your trust in the flight attendant on the airplane.

OExtremely Trust

OQuite Trust

Oslightly Trust

ONether Trust nor Distrus:

OSlighty Distrust

Qquite Distrus:

OExtremely Distrust 


\section{Control Condition}

Imagine that you are flying on a 4-hour commercial airplane flight from one major city to another. Sometime during the flight, the pilot comes on the intercom and tells you the altitude of flight and how long it will be before you land.

Please rate your trust in the automation that operates the oxygen masks.

Extremely Trust

Quite Trust

Slightly Trust

Neither Trust nor Distrust

Slightly Distrust

Quite Distrust

Extremely Distrust

Please rate your trust in the automation that operates the airplane's auto-pilot system.

Extremely Trust

Quite Trust

Slightly Trust

Neither Trust nor Distrust

Slightly Distrust

Quite Distrust

Extremely Distrust

Please rate your trust in the automation that operates the airplane's flaps.

Extremely Trust

Quite Trust

Slightly Trust

Neither Trust nor Distrust

Slightly Distrust

Quite Distrust

Extremely Distrust

Please rate your trust in the automation that operates the airplane's landing gear.

Extremely Trust

Quite Trust

Slightly Trust

Neither Trust nor Distrust

Slightly Distrust

Quite Distrust

Extremely Distrust 
Please rate your trust in the automation that operates the airplane's video screens on the backs of the seats.

Extremely Trust

Q Quite Trust

Silighty Trust

Neither Trust nor Distrust

Slighty Oistrust

Q Quite Destrust

Extremely Oistrust

Please rate your trust in the pilot that operates the airplane.

Extremely Trust

OQuite Trust

Slightly Trust

Neither Trust nor Distrust

Slightly Oistrust

Q Quite Distrust

Extremely Distrust

Please rate your trust in the co-pilot that operates the airplane.

Extremely Trust

Q Quite Trust

Silghtly Trust

Neither Trust nar Distrust

Slightly Oistrust

OQuite Destrust

Extremely Oistrust

Please rate your trust in the maintenance manager for the airline.

Extremely Trust

OQuite Trust

Slighty Trust

Neither Trust nar Distrust

Slightly Oistrust

Quite Oistrust

Extremely Distrust

Please rate your trust in the CEO of the airline manufacturer.

Extremely Trust

QQuite Trust

Slightly Trust

Neither Trust nor Distrust

Slighty Oistrust

OQuite Distrust

Extremely Oistrust

Please rate your trust in the flight attendant on the airplane.

Extremely Trust

Q Quite Trust

Slightly Trust

Neither Trust ner Oistrust

Silightly Distrust

Q Quite Destrust

Dextremely Oistrust 
Appendix B - Questions measuring affect

\section{Failure Condition}

Imagine that you are flying on a 4-hour commercial airplane flight from one major city to another. Sometime during the flight, an alarm goes off throughout the cabin and oxygen masks fall from the compartments above passenger seats. Following this, the pilot comes on the intercom and says that there was a mistake and the automation that operated the oxygen masks failed. He says that there is no actual emergency and not to worry. The pilot then tells you the altitude of flight and how long it will be before you land.

How does this make you feel?

Extremely Unfavorable

Quite Unfavorable

Slightly Unfavorable

Neutral

Slightly Favorable

Quite Favorable

Extremely Favorable

How does this make you feel?

Extremely Negative

Quite Negative

Slightly Negative

Neutral

Slightly Positive

Quite Positive

Extremely Positive

How does this make you feel?

Extremely Uncomfortable

Quite Uncomfortable

Slightly Uncomfortable

Neutral

Slightly Comfortable

Quite Comfortable

Extremely Comfortable 


\section{Control Condition}

Imagine that you are flying on a 4-hour commercial airplane flight from one major city to another. Sometime during the flight, the pilot comes on the intercom and tells you the altitude of flight and how long it will be before you land.

How does this make you feel?

Extremely Negative

Quite Negative

Slightly Negative

Neutral

Slightly Positive

Quite Positive

Extremely Positive

How does this make you feel?

Extremely Uncomfortable

Quite Uncomfortable

Slightly Uncomfortable

Neutral

Slightly Comfortable

Quite Comfortable

Extremely Comfortable

How does this make you feel?

Extremely Unfavorable

Quite Unfavorable

Slightly Unfavorable

Neutral

Slightly Favorable

Quite Favorable

Extremely Favorable 


\section{Appendix C - Variables}

\section{Stage 1 - Independent Variables Levels}

The levels of the first IV were the failure condition and the control condition. The levels of the second were the participants' countries of origin, India and the United States. The ten levels of the last IV included the five different automated aids and the five different human entities that the participant will rate. The automated aids were the oxygen masks, the auto-pilot system, the airplane's flaps, the landing gear, and the video screens on the backs of the seats. The human entities were the pilot, the co-pilot, the flight attendants, mechanics, and airline CEO. As mentioned previously, in the failure condition scenario, the oxygen mask is the automated aid that experiences the failure. 